\title{
Multiple Factors Drive Replicating Strand Composition Bias in Bacterial Genomes
}

\author{
Hai-Long Zhao ${ }^{1,2, \dagger}$, Zhong-Kui Xia ${ }^{1,2, \dagger}$, Fa-Zhan Zhang ${ }^{1,2}$, Yuan-Nong Ye ${ }^{1,2, *}$ and \\ Feng-Biao Guo $1,2, *$
}

1 Center of Bioinformatics, Key Laboratory for NeuroInformation of the Ministry of Education, School of Life Science and Technology, University of Electronic Science and Technology of China, Chengdu 610054, China; E-Mails: biohellen@gmail.com (H.-L.Z.); xiazhk@163.com (Z.-K.X.); fzzhang@cefg.cn (F.-Z.Z.)

2 Center for Information in BioMedicine, University of Electronic Science and Technology of China, Chengdu 610054, China

$\dagger$ These authors contributed equally to this work.

* Authors to whom correspondence should be addressed;

E-Mails: ynye@cefg.cn (Y.-N.Y.); fbguo@uestc.edu.cn (F.-B.G.);

Tel./Fax: +86-28-8320-2351 (Y.-N.Y.\& F.-B.G.).

Academic Editor: Patrick C. Y. Woo

Received: 27 July 2015 / Accepted: 18 September 2015 / Published: 23 September 2015

Abstract: Composition bias from Chargaff's second parity rule (PR2) has long been found
in sequenced genomes, and is believed to relate strongly with the replication process in
microbial genomes. However, some disagreement on the underlying reason for strand
composition bias remains. We performed an integrative analysis of various genomic
features that might influence composition bias using a large-scale dataset of 1111 genomes.
Our results indicate (1) the bias was stronger in obligate intracellular bacteria than in other
free-living species ( $p$-value $=0.0305)$; ( 2$)$ Fusobacteria and Firmicutes had the highest
average bias among the 24 microbial phyla analyzed; (3) the strength of selected codon
usage bias and generation times were not observably related to strand composition bias
( $p$-value $=0.3247)$; ( 4 ) significant negative relationships were found between GC content,
genome size, rearrangement frequency, Clusters of Orthologous Groups (COG) functional
subcategories A, C, I, Q, and composition bias ( $p$-values $<1.0 \times 10^{-8}$ ); $(5)$ gene density
and COG functional subcategories D, F, J, L, and V were positively related with
composition bias $\left(p\right.$-value $\left.<2.2 \times 10^{-16}\right)$; and $(6)$ gene density made the most important 
contribution to composition bias, indicating transcriptional bias was associated strongly with strand composition bias. Therefore, strand composition bias was found to be influenced by multiple factors with varying weights.

Keywords: strand composition bias; multiple factors; gene density; genomic features; COG functional category; obligate intracellular bacteria

\section{Introduction}

The DNA replication process produces two identical DNA molecules from one original DNA molecule. The leading strand is synthesized continuously in the same direction as the growing replication fork and the lagging strand is replicated by the synthesis of short and separated Okazaki fragments that are then joined together to form an integrated strand [1]. According to Chargaff's second parity rule (PR2), a single DNA strand globally has an equal percentage of base pairs $(\mathrm{A} \approx \mathrm{T}$ and $\mathrm{G} \approx \mathrm{C}$ ) when there is no strand bias caused by mutation or selection [2]. After PR2 bias caused by mutation was found between the leading and lagging strands in the echinoderm and vertebrate mitochondria genomes [3], the same phenomenon has been found in an increasing number of genomes [4-11]. These biases consistently showed that the leading strand had more $\mathrm{G}$ than $\mathrm{C}$ and, to a lesser extent more $\mathrm{T}$ than A, while in lagging strand the bias was in the opposite direction $[9,12,13]$.

Many researchers found that the strand bias was related to the replication process, because the accumulation of base mutations were caused by the asymmetric replication mechanism between the two strands $[1,2,6,14,15]$. The rule of Watson-Crick base pairing would protect cytosine from being deaminized in double-stranded DNA [16,17]. However, DNA must be separated into two single strands temporarily during replication. In single-stranded DNA, cytosine would be easier to undergo deamination and transform to thymine, which contributes towards the composition bias in genomes [16]. Researchers have found that other factors may lead to asymmetry of DNA, such as thymine dimers [18], nonsense mutations $[11,16]$, two-fold degenerated sites of cytosine $[13,19]$, and nucleotide usage in twofold as well as fourfold degenerate sites from third codon positions [20]. Other researchers suggested that the strand composition bias was associated with the transcription process [21,22]. The mutation and repair frequencies between coding and non-coding regions of genomes are different, and most genes are located on the leading strands [1,23]. Hence, considering the gene orientation bias, the transcription process also could induce composition bias between two replicating strands.

Thus, the mechanisms underlying nucleotide composition bias are still open to debate. In this work, we selected 1111 microbial genomes to study a number of factors that may affect strand composition bias, using a quantitative analysis approach.

\section{Results and Discussion}

\subsection{Composition Bias in Obligate Intracellular Bacteria}

Extremely strong strand composition bias has been reported in 11 bacteria, among which seven are obligate intracellular parasites [8]. The strong bias means that genes have significantly different base 
and codon usages between the two replicating strands [24-26]. Obligate intracellular bacteria live permanently in their hosts, which helps to protect them against some DNA damage [7]. Thus, during their long-term evolution, some DNA repair genes would have been lost and mutations would have accumulated, resulting in the strand composition bias that has been reported.

In this work, we analyzed the composition bias in obligate intracellular bacteria using a broader range of genomes than has been used previously. Among the 1111 genomes that we downloaded from the NCBI FTP site (see Section 3.1 for details), 83 bacteria were confirmed as obligate intracellular. The species names and access numbers are displayed in Table S1. The average Scorecomposition bias (see Section 3.2 for details) of the 83 obligate intracellular bacteria (0.0433) was significantly higher than that of the other bacteria (0.0362) ( $t$-test, $p$-value $=0.0305)$, and 40 of the 83 genomes were among the top scoring 258 genomes (top quarter). However, the top 10 genomes were not from obligate intracellular bacteria. Thus, the Score composition bias of obligate intracellular bacteria was stronger on the whole than that of the other species, but not always strong for an individual genome.

\subsection{Composition Bias in Different Bacterial Phyla}

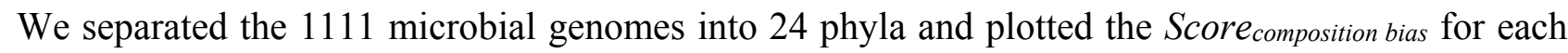

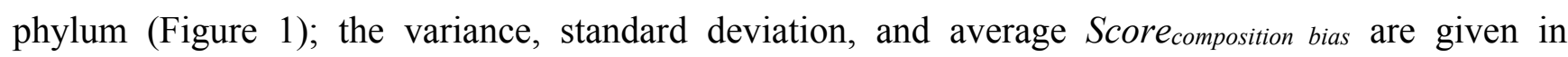
Table 1. Fusobacteria had the highest average Scorecomposition bias. They are obligately anaerobic non-spore-forming Gram-negative bacteria [27]. Firmicutes had the second highest average Score composition bias, which is in accord with a previous study that found that strand-biased gene distribution was stronger in Firmicutes than in other bacteria [28]. To explore other features that may affect composition bias at the phylum level, we compared the size, GC content, and rearrangement frequencies of the Fusobacteria and Firmicutes genomes and found that these three features were smaller than the average values for all the other bacterial genomes; however, the gene densities in these two phyla were larger than the average values for all the other bacteria (Table 2). We reconstructed the phylogenetic tree of the 24 phyla (Figure 2) and found that the Fusobacteria and Firmicutes phyla had the closest relationship. Meanwhile, they had the top two Score composition bias (0.100 and 0.071). We also found that several other clades with close relationship had similar Scorecomposition bias, such as among Gemmatimonadetes, Planctomycetes and Acidobacteria. This suggests phylogenetic relationship is one of the determinant factors of strand composition bias in bacterial genomes. 


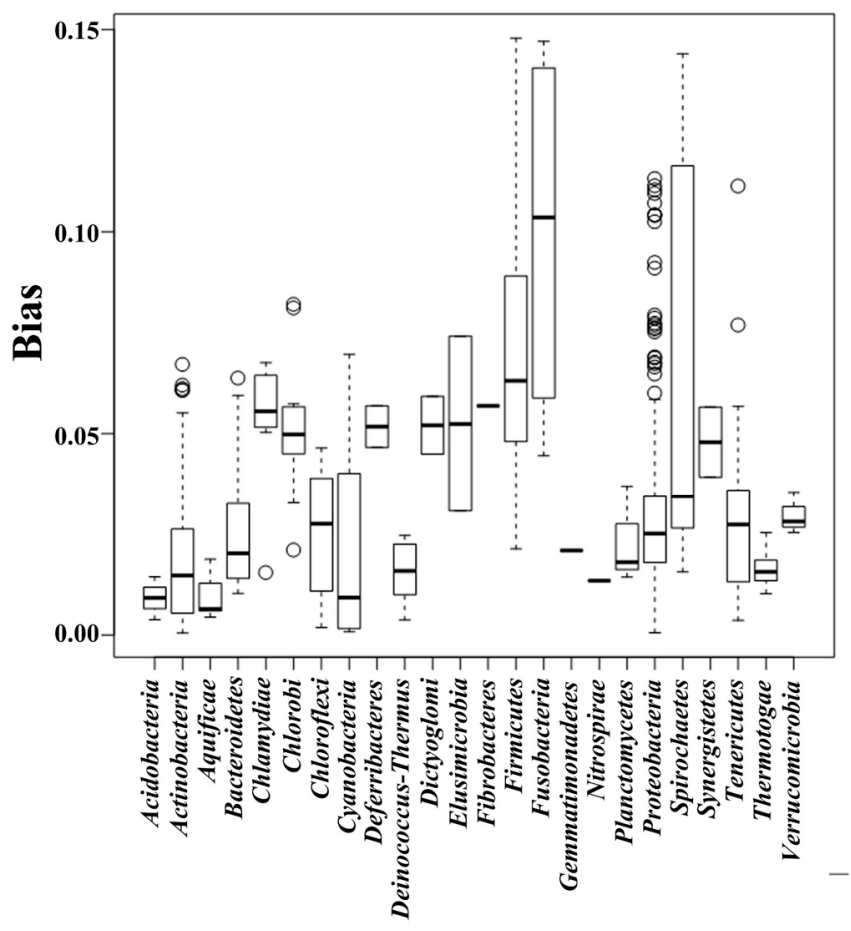

Phylum

Figure 1. Box-and-whiskers represent for composition bias of all genomes, which sorted into 24 phyla. The bottom and top of box mark the first and third quartiles, and the band inside the box denotes the median. The ends of the whiskers in each plot represent the lowest datum still within 1.5 IQR (interquartile range) of the lower quartiles, and the highest datum still within 1.5 IQR of the upper quartiles. Any data not included between the whiskers is plotted as an outlier with a small circle. This boxplot graphically depict the different bias distribution in respective phylum.

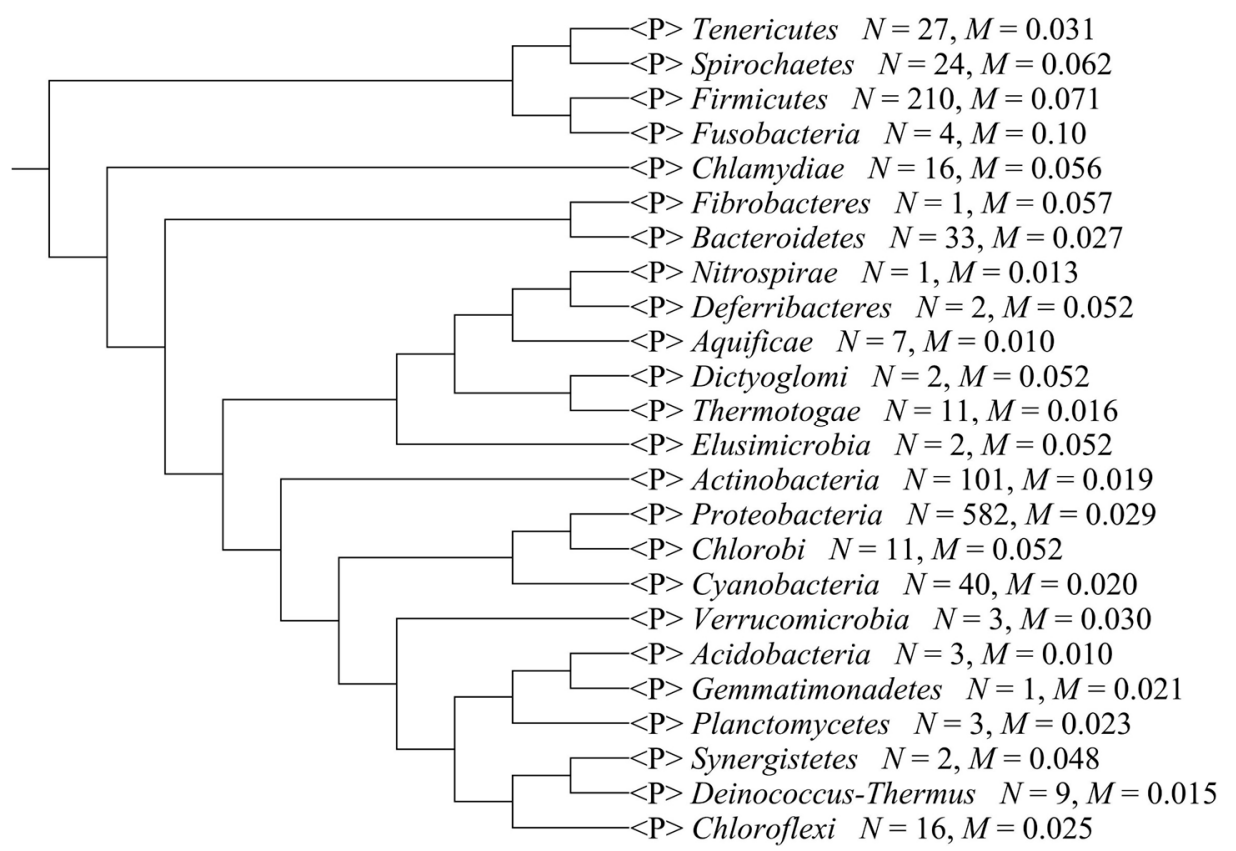

Figure 2. The phylogenetic tree of the 24 phyla. $N$ means the total strains in a phylum, $M$ means the average Scorecomposition bias in a phylum. 
Table 1. Strand composition bias for each phylum ${ }^{\text {a }}$.

\begin{tabular}{cccc}
\hline Phylum & Standard Deviation & Variance & Mean \\
\hline Acidobacteria & 0.005309 & $2.82 \times 10^{-5}$ & 0.009124 \\
Actinobacteria & 0.015749 & 0.000248 & 0.018728 \\
Aquificae & 0.005263 & $2.77 \times 10^{-5}$ & 0.00957 \\
Bacteroidetes & 0.016805 & 0.000282 & 0.027048 \\
Chlamydiae & 0.012521 & 0.000157 & 0.055526 \\
Chlorobi & 0.018046 & 0.000326 & 0.051947 \\
Chloroflexi & 0.015056 & 0.000227 & 0.024993 \\
Cyanobacteria & 0.021638 & 0.000468 & 0.019847 \\
Deferribacteres & 0.007318 & $5.36 \times 10^{-5}$ & 0.051752 \\
Deinococcus-Thermus & 0.007668 & $5.88 \times 10^{-5}$ & 0.015442 \\
Dictyoglomi & 0.010132 & 0.000103 & 0.052093 \\
Elusimicrobia & 0.030697 & 0.000942 & 0.052418 \\
Fibrobacteres & $\mathrm{NA}$ & $\mathrm{NA}$ & 0.056901 \\
Firmicutes & 0.028571 & 0.000816 & 0.071236 \\
Fusobacteria & 0.048886 & 0.00239 & 0.099682 \\
Gemmatimonadetes & $\mathrm{NA}$ & $\mathrm{NA}$ & 0.020857 \\
Nitrospirae & $\mathrm{NA}$ & $\mathrm{NA}$ & 0.013445 \\
Planctomycetes & 0.012161 & 0.000148 & 0.023082 \\
Proteobacteria & 0.017163 & 0.000295 & 0.028607 \\
Spirochaetes & 0.046978 & 0.002207 & 0.062153 \\
Synergistetes & 0.012306 & 0.000151 & 0.047907 \\
Tenericutes & 0.023255 & 0.000541 & 0.030599 \\
Thermotogae & 0.004126 & $1.70 \times 10^{-5}$ & 0.016197 \\
Verrucomicrobia & 0.005228 & $2.73 \times 10^{-5}$ & 0.029585 \\
\hline
\end{tabular}

a All genomes are grouped by phylum, NA refer to that there is only one species in this phylum. The phylum Fusobacteria owned the highest mean bias value, and the Firmicutes comes second.

Table 2. Mean value of various biological characters for each phylum ${ }^{\mathrm{a}}$.

\begin{tabular}{cccccc}
\hline Phylum & Genome Size & GC Content & Gene Density & gcRF & taRF \\
\hline Acidobacteria & $6,581,121.33$ & 0.602611 & 0.524179 & 0.546299 & 0.239179 \\
Actinobacteria & $4,434,386.26$ & 0.647473 & 0.591745 & 0.655926 & 0.5707 \\
Aquificae & $1,680,594.86$ & 0.3874153 & 0.514286 & 0.026764 & 0.090473 \\
Bacteroidetes & $3,688,038.52$ & 0.4246355 & 0.553854 & 0.035009 & 0.101365 \\
Chlamydiae & $1,265,852.44$ & 0.4046721 & 0.544713 & 0.022567 & 0.081014 \\
Chlorobi & $2,618,734.27$ & 0.5079388 & 0.583907 & 0.061015 & 0.114787 \\
Chloroflexi & $2,435,937.54$ & 0.5531583 & 0.519221 & 0.044977 & 0.063278 \\
Cyanobacteria & $3,397,176.98$ & 0.4460103 & 0.508569 & -0.33356 & -0.55797 \\
Deferribacteres & $2,728,233$ & 0.3682745 & 0.642415 & 0.012609 & 0.057666 \\
Deinococcus-Thermus & $2,411,100.11$ & 0.66285 & 0.517812 & -0.10793 & -0.12243 \\
Dictyoglomi & $1,907,773.5$ & 0.3384917 & 0.681195 & 0.01941 & 0.055101 \\
Elusimicrobia & $1,384,709.5$ & 0.3757977 & 0.726988 & 0.014904 & 0.078649 \\
Fibrobacteres & $3,842,635$ & 0.4805184 & 0.580603 & 0.047916 & 0.088216 \\
Firmicutes & $3,077,249.49$ & 0.3853 & 0.786812 & 0.020021 & 0.081354 \\
Fusobacteria & $2,680,383$ & 0.29141 & 0.72341 & 0.01046 & 0.05595 \\
\hline
\end{tabular}


Table 2. Cont.

\begin{tabular}{cccccc}
\hline Phylum & Genome Size & GC Content & Gene Density & gcRF & taRF \\
\hline Gemmatimonadetes & $4,636,964$ & 0.6427436 & 0.566455 & 0.043068 & 0.055612 \\
Nitrospirae & $2,003,803$ & 0.341289 & 0.552386 & 0.019141 & 0.07548 \\
Planctomycetes & $6,254,950$ & 0.5550987 & 0.502151 & 0.116125 & 0.138471 \\
Proteobacteria & $3,506,416.55$ & 0.5337785 & 0.569934 & 0.067462 & 0.135439 \\
Spirochaetes & $1,702,653.17$ & 0.3721947 & 0.600467 & 0.021591 & 0.121083 \\
Synergistetes & $1,914,533$ & 0.5454971 & 0.75006 & 0.023406 & 0.050368 \\
Tenericutes & $892,007.889$ & 0.2794737 & 0.665323 & -0.02018 & -0.08702 \\
Thermotogae & $1,976,742.36$ & 0.4028872 & 0.54724 & 0.024232 & 0.083806 \\
Verrucomicrobia & $3,998,507$ & 0.5480856 & 0.51413 & 0.093882 & 0.10771 \\
Mean & $3,329,265.48$ & 0.4952767 & 0.612158 & 0.092191 & 0.127667 \\
\hline
\end{tabular}

${ }^{a}$ Genome size, GC content and rearrangement frequency of Fusobacteria and Firmicutes are all smaller than average of each trait for all genomes, but the opposite was true for the gene density.

\subsection{Composition Bias in Genomes with Different $S$ Values}

Selection and mutation are two primary factors that generate bias in species' genomes during evolution. These two factors may generate biases that partially counteract each other. An $S$ value can be used to measure the strength of codon usage bias as an indicator of selection bias [29]. Replicating strand composition bias can be considered to represent mutation bias. Thus, we used the $S$ values for 80 bacterial genomes that were reported by Sharp et al. [29] to study the correlation between them and the Score composition bias of the same 80 genomes. We found that there was no significant correlation between them (Spearman's correlation, $\rho=-0.08604675$, $p$-value $=0.3247$ ). Hence, we suggest that selection and mutation may influence genome bias by different mechanisms; therefore, codon usage bias may counteract strand composition bias.

\subsection{Composition Bias in Genomes with Different Generation Times}

Microbial generation times range from a few minutes to several weeks and are affected by evolutionary factors such as environment stability, nutrient availability, and community diversity. Vieira-Silva and Rocha found that codon usage bias was correlated with growth rates [30]. Hence, we

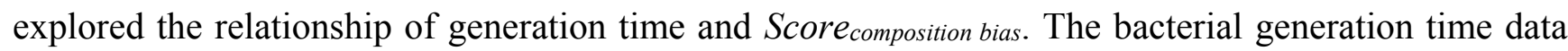
were extracted from of the paper by Vieira-Silva and Rocha [30]. Our result indicated that generation time also was not significantly related with Scorecomposition bias (Spearman's correlation, $\rho=-0.1457365, p$-value $=0.1021)$. That may be the same as the reason mentioned on the $S$ value.

\subsection{Composition Bias in Genomes with Different Genome Sizes}

The average sizes of the genomes in the Fusobacteria and Firmicutes phyla are smaller than average sizes of the genomes in all the bacterial phyla examined. We found that a significantly negative correlation existed between genome size and Scorecomposition bias (Spearman's correlation, $\rho=-0.2508015, p$-value $<2.2 \times 10^{-16}$ ). This finding is similar to the results of Guo and Ning [7] who found that the genome sizes of 11 bacteria with extremely strong strand composition biases were all 
smaller than $2000 \mathrm{~kb}$. Guo and Ning speculated that the repair mechanism might be inefficient in small bacterial genomes that had undergone reductive evolution [7]. Additionally, mutation pressure may be insufficient to surpass translational selection in larger genomes.

\subsection{Composition Bias in Genomes with Different Gene Densities of the Leading Strand}

With the availability of a large number of complete genome sequences, it has become increasing clear that the unequal distribution of genes between leading and lagging strands varies widely among different species. Numerous studies have shown that genes are generally preferentially located on the leading strand [31-34], which may be explained by the polymerase collision avoidance model [1].

We calculated the density of leading strand genes for all 1111 genomes. Our correlation analysis showed that gene density was highly positively correlated with Score composition bias (Spearman's correlation, $\rho=0.6273871, p$-value $<2.2 \times 10^{-16}$ ). This result could be caused by DNA replication-associated mutation bias during the transcription process in which DNA decomposes into single strands. However, the DNA mutation or repair rates were quite different between transcribed and non-transcribed strands. Because most protein-coding genes are located on the leading strand, the two replication strands can have extremely different compositions [21]. Thus, the asymmetric transcription process is likely to have a major impact on the composition bias between the two replication strands.

\subsection{Composition Bias in Genomes with Different GC Contents}

GC content is the percentage of guanine and cytosine base pairs in a DNA sequence. The GC content of bacterial genomes ranges from about $20 \%$ to $70 \%$ [35]. We investigated the correlation between GC content and Score composition bias and found that a significantly negative correlation existed between them (Spearman's correlation, $\rho=-0.5026315, p$-value $<2.2 \times 10^{-16}$ ). It may be explained that genomes with high GC content will generate fewer mutations than those with low GC content [36]. However, this would inspire us that the replicating strand composition bias is caused by a complex set of factors.

\subsection{Composition Bias in Genomes with Different Recombination Rates}

Chromosomal recombination occurs as a result of deletions, duplications, inversions, and translocations in native chromosomes. Rocha [1] has shown that the recombination rate is related to strand composition bias, and has suggested that codon usage separation may be caused by low recombination rates in some obligate intracellular parasites. Wei and Guo confirm this suggestion in 11 obligate intracellular bacteria with strong strand composition bias using the Z-curve method [24].

Here, we explored this issue in the 1111 genomes. The recombination rates $(t a R F, g c R F)$ of each genome were calculated as described in Section 3.3. Then, the correlations between Scorecomposition bias and both $\operatorname{taRF}$ and $g c R F$ were estimated for all the genomes. We found that $t a R F$ and $g c R F$ were both negatively associated with Scorecomposition bias (Spearman's correlations, $\rho_{g c R F}=-0.3746862$, $\rho_{t a R F}=-0.2916134$, both $p$-values $<2.2 \times 10^{-16}$ ). 
Rocha suggested that frequent chromosomal recombination would reduce strand composition bias [1]. The base distribution in any one strand is accordant; that is, if $\mathrm{G}>\mathrm{C}$ in a particular region, then a similar base distribution also will be found in other regions of the same chromosome. However, recombination would break the accordance and reduce strand composition bias.

\subsection{Composition Bias in Different COG Functional Categories}

To determine whether gene function has an impact on strand composition bias, we explored the relationship between Clusters of Orthologous Groups (COG) functional categories and composition bias for the first time.

\subsubsection{Percentage of Gene Number for Each COG Functional Subcategory}

To explore the influence of each COG subcategory on composition bias, the correlation between the percentage of each COG functional subcategory (pCOGi; see Section 3.4 for details) and the corresponding Scorecomposition bias was analyzed for each genome. The results, summarized in Table 3, were considered as statistically significant if the $p$-value was $<1.0 \times 10^{-8}$. Based on this cutoff value, the pCOGs of the A, C, I, and Q subcategories were negatively related to Scorecomposition bias, and the D, F, J, L, and V subcategories showed positive correlations to Scorecomposition bias.

Klasson and Andersson have studied gene function and composition bias [37]. They found that strong asymmetric mutation bias in endosymbiont genomes caused them to lack replication restart genes (subcategory L). Guo and Ning reported that genes associated with replication initiation and re-initiation such as $m u t H$, dnaT and fis were absent in 11 obligate intracellular bacteria genomes with extreme strand composition bias [7]. However, we detected some replication initiation and re-initiation genes based on our analysis of the 1111 genomes, which indicated that COG subcategory L and composition bias was positively correlated. This is an interesting finding that we will further explore in Section 2.9.2. Rocha and Danchin [38] reported some obligate parasite bacteria with strong composition bias in which genes associated with energy metabolism were absent. This finding is mostly accord with our result that the metabolism-related genes (subcategories C, I, and Q) were all negatively correlated with composition bias, except those in subcategory F.

Table 3. The correlation of each Clusters of Orthologous Groups (COG) functional subcategory and strand composition bias.

\begin{tabular}{|c|c|c|c|}
\hline & COG Functional Category & $p$ Value & Correlation \\
\hline \multicolumn{4}{|c|}{ Information Storage and Processing } \\
\hline $\mathrm{J}$ & Translation, ribosomal structure and biogenesis ${ }^{\mathrm{P}}$ & $8.11 \times 10^{-32}$ & 0.341886 \\
\hline A & RNA processing and modification ${ }^{\mathrm{N}}$ & $2.44 \times 10^{-13}$ & -0.21728 \\
\hline K & Transcription & 0.099239 & -0.04948 \\
\hline $\mathrm{L}$ & Replication, recombination and repair ${ }^{\mathrm{P}}$ & $1.01 \times 10^{-8}$ & 0.170797 \\
\hline $\mathrm{B}$ & Chromatin structure and dynamics & 0.002404 & -0.09097 \\
\hline \multicolumn{4}{|c|}{ Cellular Processes and Signaling } \\
\hline $\mathrm{D}$ & Cell cycle control, cell division, chromosome partitioning ${ }^{\mathrm{P}}$ & $1.05 \times 10^{-45}$ & 0.407564 \\
\hline Y & Nuclear structure & 0.222949 & 0.036592 \\
\hline $\mathrm{V}$ & Defense mechanisms ${ }^{\mathrm{P}}$ & $3.93 \times 10^{-14}$ & 0.224269 \\
\hline
\end{tabular}


Table 3. Cont.

\begin{tabular}{|c|c|c|c|}
\hline & COG Functional Category & $p$ Value & Correlation \\
\hline $\mathrm{T}$ & Signal transduction mechanisms & $1.77 \times 10^{-7}$ & -0.15589 \\
\hline \multirow[t]{2}{*}{ M } & Cell wall/membrane/envelope biogenesis & 0.609835 & -0.01533 \\
\hline & Cellular Processes and Signaling & & \\
\hline $\mathrm{N}$ & Cell motility & 0.198305 & 0.038623 \\
\hline Z & Cytoskeleton & 0.006632 & -0.0814 \\
\hline $\mathrm{W}$ & Extracellular structures & 0.901043 & -0.00373 \\
\hline $\mathrm{U}$ & Intracellular trafficking, secretion, and vesicular transport & 0.908091 & 0.003467 \\
\hline \multirow[t]{2}{*}{$\mathrm{O}$} & Posttranslational modification, protein turnover, chaperones & 0.188347 & -0.0395 \\
\hline & Metabolism & & \\
\hline $\mathrm{C}$ & Energy production and conversion ${ }^{\mathrm{N}}$ & $4.51 \times 10^{-11}$ & -0.1959 \\
\hline G & Carbohydrate transport and metabolism & 0.193919 & 0.039003 \\
\hline $\mathrm{E}$ & Amino acid transport and metabolism & 0.417676 & -0.02434 \\
\hline $\mathrm{F}$ & Nucleotide transport and metabolism ${ }^{\mathrm{P}}$ & $5.99 \times 10^{-39}$ & 0.377498 \\
\hline $\mathrm{H}$ & Coenzyme transport and metabolism & 0.01405 & 0.073666 \\
\hline I & Lipid transport and metabolism ${ }^{\mathrm{N}}$ & $1.22 \times 10^{-19}$ & -0.26737 \\
\hline $\mathrm{P}$ & Inorganic ion transport and metabolism & 0.081681 & -0.05226 \\
\hline $\mathrm{Q}$ & Secondary metabolites biosynthesis, transport and catabolism ${ }^{\mathrm{N}}$ & $6.65 \times 10^{-40}$ & -0.38194 \\
\hline
\end{tabular}

$\mathrm{N}$ denotes significantly negative correlation between subcategories and composition bias. $\mathrm{P}$ denotes significantly positive correlation between subcategories and composition bias.

\subsubsection{Proportion of Replication and Repair Genes}

The correlation between subcategory $\mathrm{L}$ and composition bias that we obtained is opposite to what has been found previously. To explore this result further, we collected the replication and repair genes from the KEGG pathway database and divided then into the 10 subtypes (for details see Section 3.7) based on their functions. The correlations between the percentage genes under each subtype and the Scorecomposition bias are shown in Table 4. The gene subtypes were all positively related to composition bias, and the excision and mismatch repair subtype had the highest correlation. We suspect that genomes with strong composition bias may have generated more repair genes to balance the composition bias during evolution. However, the cause-and-effect relationship between repair genes and composition bias is not still clear; that is, which is the cause and which is the effect.

\subsubsection{Average Value of Times between Strong-Biased Group and Weak-Biased Group for Each Functional Subcategory}

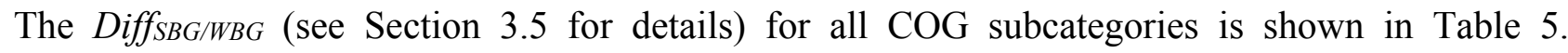
Subcategory D had the highest value (5.709 among all the subcategories, which indicated that genes involved in cell cycle control, cell division, and chromosome partitioning were present in significant numbers in the strong-biased genomes (i.e., the genomes with three top 555 Scorecomposition bias values). This result is in accordance with Lin et al. [39] who found that only some essential COG subcategories were situated preferentially on the leading strand and that subcategory D genes showed the most significant bias among 10 strand-biased classifications. Furthermore, both the strong-biased COG groups (SCOGs) and weak-biased COG groups (WCOGs) in all 1111 genomes were significantly 
related to Score composition bias (Spearman's correlation, $\rho \mathrm{SCOG}=0.51473$ and $\rho \mathrm{WCOG}=-0.65945$, both $p$-values $<2.2 \times 10^{-16}$ ). We suggest that although the essential subcategories are similar in number in the genomes, they tend to be located on the leading strand, resulting in strong composition bias. For small genomes, the percentages of essential subcategories are higher than for large genomes, hence leading to stronger composition bias in small genomes.

Table 4. Average value of discrepant times (AVDT) between strong-biased group and week-biased group for each functional subcategory in descending order.

\begin{tabular}{cccc}
\hline COG & AVDT & COG & AVDT \\
\hline D & 5.709197 & $\mathrm{C}$ & 1.086021 \\
$\mathrm{~K}$ & 3.415376 & $\mathrm{H}$ & 1.053758 \\
$\mathrm{~N}$ & 2.848684 & $\mathrm{~F}$ & 1.046122 \\
$\mathrm{~T}$ & 2.229241 & $\mathrm{~V}$ & 1.02066 \\
$\mathrm{M}$ & 2.181872 & $\mathrm{E}$ & 0.99786 \\
$\mathrm{O}$ & 2.089135 & $\mathrm{I}$ & 0.936222 \\
$\mathrm{U}$ & 2.013089 & $\mathrm{P}$ & 0.914553 \\
$\mathrm{G}$ & 1.472415 & $\mathrm{~A}$ & 0.864394 \\
$\mathrm{~L}$ & 1.363586 & $\mathrm{Z}$ & 0.775298 \\
$\mathrm{~B}$ & 1.266486 & $\mathrm{Q}$ & 0.64794 \\
$\mathrm{~J}$ & 1.23429 & $\mathrm{~W}$ & 0.6 \\
\hline
\end{tabular}

Table 5. Relationship between each type of replication and repair genes and composition bias.

\begin{tabular}{lccc}
\hline Pathway & Function & $\boldsymbol{p}$ Value & Correlation \\
\hline ko03030 & DNA replication & $3.69 \times 10^{-10}$ & 0.18656 \\
ko03032 & DNA replication proteins & $6.70 \times 10^{-9}$ & 0.172841 \\
ko03036 & Chromosome and associated proteins & $3.28 \times 10^{-7}$ & 0.152472 \\
ko03400 & DNA repair and recombination proteins & $6.73 \times 10^{-10}$ & 0.183808 \\
ko03410 & Base excision repair & $2.11 \times 10^{-6}$ & 0.141724 \\
ko03420 & Nucleotide excision repair & $4.15 \times 10^{-12}$ & 0.2059713 \\
ko03430 & Mismatch repair & $9.39 \times 10^{-12}$ & 0.2025802 \\
ko03440 & Homologous recombination & $1.16 \times 10^{-10}$ & 0.191753 \\
ko03450 & Non-homologous end-joining & 0.926821 & 0.002759 \\
ko03460 & Fanconi anemia pathway & 0.002531 & 0.090509 \\
\hline
\end{tabular}

\subsection{Conjoint Analysis of Multiple Factors and Composition Bias by Principal Component Regression}

We determined the independent contribution of each genomic feature to composition bias by principal component regression. Here, we selected only the features that were significantly related with strand composition bias ( $p$-values $<1.0 \times 10^{-8}$ ). The replication and repair genes were not considered separately because they belong to COG subcategory $\mathrm{L}$. The respective contribution is presented in detail in Table 6 . The results show that among the whole contribution $\left(R^{2}=0.5104\right)$ of all the features, gene density $\left(R^{2}=0.064778\right)$ made the most contribution to strand composition bias. Thus, gene orientation bias was the primary factor that influenced base composition among the biological features tested. 
Table 6. Principal component regression analysis of various genomic features ${ }^{\text {a }}$.

\begin{tabular}{ccccccccc}
\hline $\begin{array}{c}\text { Genomic } \\
\text { Features }\end{array}$ & $\begin{array}{c}\text { Genome } \\
\text { Size }\end{array}$ & $\begin{array}{c}\text { Gene } \\
\text { Density }\end{array}$ & GC Content & gcRF & taRF & SCOGs & WCOGs & A \\
\hline$R^{2}$ & 0.0558 & 0.0648 & 0.0391 & 0.0004 & 0.0003 & 0.0332 & 0.0326 & 0.0122 \\
$\begin{array}{c}\text { Genomic } \\
\text { features }\end{array}$ & $\mathrm{C}$ & $\mathrm{D}$ & $\mathrm{F}$ & $\mathrm{I}$ & $\mathrm{J}$ & $\mathrm{L}$ & $\mathrm{Q}$ & $\mathrm{V}$ \\
$R^{2}$ & 0.0634 & 0.0348 & 0.0272 & 0.0238 & 0.0299 & 0.0371 & 0.0262 & 0.0297 \\
\hline
\end{tabular}

${ }^{\text {a }}$ Detail values for each of the genomic features and strand composition bias are listed in Table S2.

\section{Experimental Section}

\subsection{Data Source}

We retrieved 1111 bacterial genome sequences from the NCBI FTP site in September 2010. Among them, 76 bacteria had multiple strains and hence the 1111 bacteria belonged to only 703 species. We used all sequenced bacterial genomes at that time, rather than sampling the genomic data to analyze.

The origin and terminus of DNA replications were obtained from the Doric database [40] in July 2011. This information was used to separate genes onto leading and lagging strands.

The genes related to DNA repair and replications were extracted from the KEGG Pathway database [41] in April 2013.

\subsection{Computation of Strand Composition Bias}

Strand composition bias of a whole genome was obtained as:

$$
\text { Score } \text { Composition Bias }=\frac{|G-C|+|T-A|}{\text { Chromosome Length }}
$$

where $G, C, T$, and $A$ are the numbers of corresponding bases in leading strands. According to the principle of complementary base pairing, strand composition bias in lagging strands is equal to that of the leading strand.

\subsection{Computation of Counteracting Effect of Recombination}

Strand composition bias was measured by the mean value of $G-C+T-A$. Recombination may change the natural order of nucleotides, so to counteract some usual bias and finally lower the strength of the whole bias, we introduced two values, $g c R F$ and $t a R F$, to roughly reflect this effect of recombination. $g c R F$ was calculated as:

$$
\begin{gathered}
\overline{\text { gcBias }}=\frac{\sum_{i=1}^{N} \frac{G_{i}-C_{i}}{L_{i}}}{N} \\
g c R F=\frac{\sum_{i=1}^{N}\left(\frac{G_{i}-C_{i}}{L_{i}}-\overline{\text { gcBias }}\right)^{2}}{(N-1) \times \overline{\text { gcBias }}}
\end{gathered}
$$

and $\operatorname{taRF}$ and was calculated as: 


$$
\begin{gathered}
\overline{\text { taBias }}=\frac{\sum_{i=1}^{N} \frac{T_{i}-A_{i}}{L_{i}}}{N} \\
\operatorname{taRF}=\frac{\sum_{i=1}^{N}\left(\frac{T_{i}-A_{i}}{L_{i}}-\overline{\text { taBias }}\right)^{2}}{(N-1) \times \overline{\text { taBias }}}
\end{gathered}
$$

where $G_{i}, C_{i}, T_{i}$, and $A_{i}$ are the numbers of corresponding bases of the $i^{t h}$ leading strand gene; $L_{i}$ is the length of the corresponding gene; and $N$ is the total number of genes in the leading strand. Usually, the higher the two values are, the higher the frequency of counteracting recombination occurs.

\subsection{Computation of the Percentage of Each COG Functional Subcategory}

The percentage of each COG functional subcategory (pCOG) was calculated as:

$$
p C O G_{i}=\frac{N_{C O G} i}{N_{C O G}} \quad i=A \sim Z, \text { except } \quad R, S, X
$$

where $i$ is the $i^{t h}$ subcategory and $N_{C O G i}$ is the number of genes with the $i^{\text {th }}$ subcategory in a genome. $N_{C O G}$ is the total number of genes within all the COG subcategories.

\subsection{Computation of Average Value of Differences between Strong-Biased Group and Weak-Biased Group for Each Functional Subcategory}

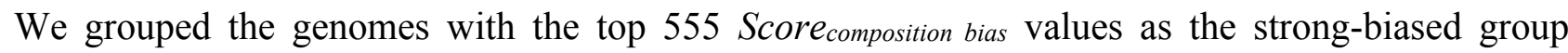
(SBG), and the remaining genomes as the weak-biased group (WBG) and count the number of genes in each COG subcategory for all the species in each group separately. For each COG, we defined an

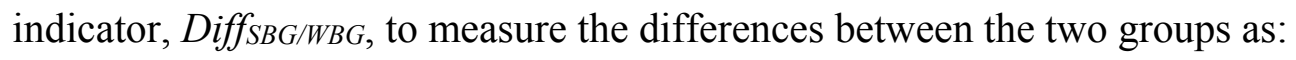

$$
\operatorname{Diff} f_{S B G} / W B G=\frac{N_{S B G}}{N_{W B G}}
$$

where $N_{S B G}$ is the number of genes in each COG subcategory in the SBG, and $N_{W B G}$ is the number of genes in each COG subcategory in the WBG.

Finally, we defined another indicator, Diff ${ }_{C O G}$, for each COG functional subcategory as:

$$
\operatorname{Diff}_{C O G} i=\frac{\sum_{j=1}^{N} \operatorname{Diff}_{S B G} / W B G j}{N} \quad i=A \sim Z, \text { except } \quad R, S, X
$$

where $i$ is the $i^{\text {th }}$ subcategory of the 23 COG functional subcategories; $j$ is the $j^{\text {th }}$ gene in $i^{\text {th }}$ subcategory; and $N$ is the total number of genes in $i^{\text {th }}$ subcategory.

\subsection{Proportion of SCOGs and WCOGs}

Subcategories with Diff COG $>5$ were defined as strong-biased COG groups (SCOGs), and subcategories with $\operatorname{Diff}_{C O G}<0.2$ were defined as weak-biased COG groups (WCOGs). Then, the proportions of SCOGs and WCOGs in each genome were calculated. 


\subsection{Proportion of Replication and Repair Genes}

We download the genes associated with replication and repair from the Kyoto Encyclopedia of Genes and Genomes (KEGG) Pathway database [41]. Ten pathways are classified under replication and repair; namely, DNA replication, DNA replication proteins, chromosome and associated proteins, DNA repair and recombination proteins, base excision repair, nucleotide excision repair, mismatch repair, homologous recombination, non-homologous end-joining, and Fanconi anemia pathway. Then, we computed the proportion of genes associated with each classification in each genome.

\subsection{Statistical Analyses}

The correlations between various genomic features and the strand composition bias were measured by Spearman's rank correlation coefficient, which is a nonparametric measure of statistical dependence between two factors. It uses a monotonic function to assess how well the relationship between two variables. Rho of Spearman's rank correlation is used to reflect the intensity of correlation between variables of statistical indicators and the absolute value of rho reflects the relative significance between two variables. For example, a rho value of -0.14 is less significant than a rho value of -0.25 . The $p$-value of Spearman's correlation is used for measuring significance of correlation between two variables. In this work, it is considered a significant correlation if the $p$-value $<0.05$. The independent contribution of each feature to the bias was confirmed statistically by principal component regression analysis. All statistical analyses were conducted using the freely available R package (https://cran.r-project.org/).

\section{Conclusions}

Strand composition bias has been reported in different genomes over many years. The bias might be driven by multiple factors. In this work, we explored the relationship between strand composition bias and various genomic features. The results show that multiple factors are related to replication strand composition bias. Together, these factors play a major role and our principal component regression analysis showed that their contribution to replication strand composition bias accounted for over $50 \%$ of the bias. Gene orientation bias had the highest independent contribution, which indicates that the transcription process is likely to have a major impact on the composition bias between two replication strands. For most of the factors, we, for the first time, quantitatively measured their contribution to strand composition bias. Thus, so far, this study is the first integrative analysis of strand composition bias in prokaryotes. The results will help understand the underlying mechanisms of how such bias is generated.

\section{Supplementary Materials}

Supplementary materials can be found at http://www.mdpi.com/1422-0067/16/09/23111/s1.

\section{Acknowledgments}

This work was supported by the National Natural Science Foundation of China (grant numbers 31071109 and 31470068), the Fundamental Research Funds for the Central Universities of China 
(grant number ZYGX2013J101), and the Sichuan Youth Science and Technology Foundation of China (grant number 2014JQ0051).

\section{Author Contributions}

Conceived and designed the experiments: Feng-Biao Guo and Yuan-Nong Ye. Performed the experiments: Hai-Long Zhao and Zhong-Kui Xia. Analyzed the data: Zhong-Kui Xia and Fa-Zhan Zhang. Wrote the manuscript: Yuan-Nong Ye and Hai-Long Zhao. Polished the manuscript: Feng-Biao Guo and Zhong-Kui Xia.

\section{Conflicts of Interest}

The authors declare no conflict of interest.

\section{References}

1. Rocha, E.P. The replication-related organization of bacterial genomes. Microbiology 2004, 150, 1609-1627.

2. Frank, A.C.; Lobry, J.R. Asymmetric substitution patterns: A review of possible underlying mutational or selective mechanisms. Gene 1999, 238, 65-77.

3. Asakawa, S.; Kumazawa, Y.; Araki, T.; Himeno, H.; Miura, K.; Watanabe, K. Strand-specific nucleotide composition bias in echinoderm and vertebrate mitochondrial genomes. J. Mol. Evol. 1991, 32, 511-520.

4. Lobry, J.R. Asymmetric substitution patterns in the two DNA strands of bacteria. Mol. Biol. Evol. 1996, 13, 660-665.

5. Xia, X. DNA replication and strand asymmetry in prokaryotic and mitochondrial genomes. Curr. Genom. 2012, 13, 16-27.

6. Necsulea, A.; Lobry, J.R. A new method for assessing the effect of replication on DNA base composition asymmetry. Mol. Biol. Evol. 2007, 24, 2169-2179.

7. Guo, F.-B.; Ning, L.-W. Strand-Specific Composition Bias in Bacterial Genomes; INTECH Open Access Publisher: Rijeka, Croatia, 2011.

8. Guo, F.B. Replicating strand asymmetry in bacterial and eukaryotic genomes. Curr. Genom. 2012, 13, 2-3.

9. Arakawa, K.; Tomita, M. Measures of compositional strand bias related to replication machinery and its applications. Curr. Genom. 2012, 13, 4-15.

10. Lin, Q.; Cui, P.; Ding, F.; Hu, S.; Yu, J. Replication-associated mutational pressure (RMP) governs strand-biased compositional asymmetry (SCA) and gene organization in animal mitochondrial genomes. Curr. Genom. 2012, 13, 28-36.

11. Khrustalev, V.V.; Barkovsky, E.V. A blueprint for a mutationist theory of replicative strand asymmetries formation. Curr. Genom. 2012, 13, 55-64.

12. Arakawa, K.; Suzuki, H.; Tomita, M. Quantitative analysis of replication-related mutation and selection pressures in bacterial chromosomes and plasmids using generalised GC skew index. BMC Genom. 2009, 10, 640. 
13. Khrustalev, V.V.; Barkovsky, E.V. Study of completed archaeal genomes and proteomes: Hypothesis of strong mutational at pressure existed in their common predecessor. Genom. Proteom. Bioinform. 2010, 8, 22-32.

14. Lobry, J.R.; Sueoka, N. Asymmetric directional mutation pressures in bacteria. Genome Biol. 2002, 3, RESEARCH0058.

15. Khrustalev, V.V.; Barkovsky, E.V. "Protoisochores" in certain archaeal species are formed by replication-associated mutational pressure. Biochimie 2011, 93, 160-167.

16. Khrustalev, V.V.; Barkovsky, E.V. The probability of nonsense mutation caused by replication-associated mutational pressure is much higher for bacterial genes from lagging than from leading strands. Genomics 2010, 96, 173-180.

17. Beletskii, A.; Bhagwat, A.S. Transcription-induced mutations: Increase in c to t mutations in the nontranscribed strand during transcription in escherichia coli. Proc. Natl. Acad. Sci. USA 1996, 93, 13919-13924.

18. Cordeiro-Stone, M.; Nikolaishvili-Feinberg, N. Asymmetry of DNA replication and translesion synthesis of UV-induced thymine dimers. Mutat. Res. Fundam. Mol. Mech. Mutagen. 2002, 510, 91-106.

19. Khrustalev, V.V.; Barkovsky, E.V. The level of cytosine is usually much higher than the level of guanine in two-fold degenerated sites from third codon positions of genes from simplex- and varicelloviruses with $\mathrm{G}$ plus $\mathrm{C}$ higher than 50\%. J. Theor. Biol. 2010, 266, 88-98.

20. Khrustalev, V.; Barkovsky, E. Bioinformatical approaches for studies on replication-associated and transcription-associated mutational pressure, interpretations and applications. Adv. Genet. Res. 2011, 6, 1-108.

21. Francino, M.P.; Ochman, H. Strand asymmetries in DNA evolution. Trends Genet. 1997, 13, 240-245.

22. Nikolaou, C.; Almirantis, Y. A study on the correlation of nucleotide skews and the positioning of the origin of replication: Different modes of replication in bacterial species. Nucleic Acids Res. 2005, 33, 6816-6822.

23. Rocha, E.P. The organization of the bacterial genome. Annu. Rev. Genet. 2008, 42, 211-233.

24. Wei, W.; Guo, F.B. Strong strand composition bias in the genome of ehrlichia canis revealed by multiple methods. Open Microbiol. J. 2010, 4, 98-102.

25. Guo, F.B.; Yu, X.J. Separate base usages of genes located on the leading and lagging strands in chlamydia muridarum revealed by the $Z$ curve method. BMC Genom. 2007, 8, 366.

26. Guo, F.B.; Yuan, J.B. Codon usages of genes on chromosome, and surprisingly, genes in plasmid are primarily affected by strand-specific mutational biases in lawsonia intracellularis. DNA Res. 2009, 16, 91-104.

27. Bennett, K.W.; Eley, A. Fusobacteria: New taxonomy and related diseases. J. Med. Microbiol. 1993, 39, 246-254.

28. Hu, J.; Zhao, X.; Yu, J. Replication-associated purine asymmetry may contribute to strand-biased gene distribution. Genomics 2007, 90, 186-194.

29. Sharp, P.M.; Bailes, E.; Grocock, R.J.; Peden, J.F.; Sockett, R.E. Variation in the strength of selected codon usage bias among bacteria. Nucleic Acids Res. 2005, 33, 1141-1153. 
30. Vieira-Silva, S.; Rocha, E. The systemic imprint of growth and its uses in ecological (meta) genomics. PLoS Genet. 2010, 6, e1000808.

31. McLean, M.J.; Wolfe, K.H.; Devine, K.M. Base composition skews, replication orientation, and gene orientation in 12 prokaryote genomes. J. Mol. Evol. 1998, 47, 691-696.

32. Blattner, F.R.; Plunkett, G., 3rd; Bloch, C.A.; Perna, N.T.; Burland, V.; Riley, M.; Collado-Vides, J.; Glasner, J.D.; Rode, C.K.; Mayhew, G.F.; et al. The complete genome sequence of Escherichia coli K-12. Science 1997, 277, 1453-1462.

33. Rocha, E.P. Is there a role for replication fork asymmetry in the distribution of genes in bacterial genomes? Trends Microbiol. 2002, 10, 393-395.

34. Karlin, S. Bacterial DNA strand compositional asymmetry. Trends Microbiol. 1999, 7, 305-308.

35. Hildebrand, F.; Meyer, A.; Eyre-Walker, A. Evidence of selection upon genomic GC-content in bacteria. PLoS Genet. 2010, 6, e1001107.

36. Paul, S.; Million-Weaver, S.; Chattopadhyay, S.; Sokurenko, E.; Merrikh, H. Accelerated gene evolution through replication-transcription conflicts. Nature 2013, 495, 512-515.

37. Klasson, L.; Andersson, S.G. Strong asymmetric mutation bias in endosymbiont genomes coincide with loss of genes for replication restart pathways. Mol. Biol. Evol. 2006, 23, 1031-1039.

38. Rocha, E.P.; Danchin, A. Base composition bias might result from competition for metabolic resources. Trends Genet. 2002, 18, 291-294.

39. Lin, Y.; Gao, F.; Zhang, C.T. Functionality of essential genes drives gene strand-bias in bacterial genomes. Biochem. Biophys. Res. Commun. 2010, 396, 472-476.

40. Gao, F.; Luo, H.; Zhang, C.T. Doric 5.0: An updated database of oric regions in both bacterial and archaeal genomes. Nucleic Acids Res. 2013, 41, D90-D93.

41. Kanehisa, M.; Goto, S.; Kawashima, S.; Okuno, Y.; Hattori, M. The KEGG resource for deciphering the genome. Nucleic Acids Res. 2004, 32, D277-D280.

(C) 2015 by the authors; licensee MDPI, Basel, Switzerland. This article is an open access article distributed under the terms and conditions of the Creative Commons Attribution license (http://creativecommons.org/licenses/by/4.0/). 